\title{
RESEARCH HIGHLIGHT Taking action: empathy and social interaction in rats
}

\author{
Marco Venniro (D) and Sam A. Golden (D) ${ }^{2}$ \\ Neuropsychopharmacology (2020) 45:1081-1082; https://doi.org/10.1038/s41386-019-0596-0
}

Imagine you are walking and notice a person crying on the sidewalk visibly in distress. This individual is not familiar to you and has not requested any assistance, so you understandably hesitate to approach. A likely question you may ask yourself in that moment of indecision is whether you would like a stranger to offer support if you found yourself in a similar situation. Do you ultimately stop to offer emotional support? The ability to understand the circumstances of someone other than yourself is defined as empathy. Empathy is a complex behavior that facilitates the formation of social connections through interpersonal socialization and aid. A lack of empathy can contribute to the psychopathology of several neuropsychiatric diseases, including autism spectrum disorder and substance use disorder. Therefore, it is crucial to understand the psychological processes and neurobiological substrates of empathic behavior.

Empathy requires a nuanced understanding of the interaction between emotions and perceptions and has been challengingeven controversial-to model in the preclinical laboratory setting. Early studies from the 1950-1960s established that rats will exhibit emotional reactions when witnessing a conspecific in distress $[1,2]$. More recent work have extended these observations by showing that rats will release a distressed conspecific from restraint [3] or a water-filled compartment [4]. However, these procedures have not disambiguated the role of direct social interaction from indirect empathic behavior; both the distressed target and the empathic observer interact in the same environment after rescue, leading to the possibility that rewarding social interaction and contact is what drives rodent empathy $[5,6]$. Thus, in this issue of Neuropsychopharmacology, Cox and Reichel [7] introduce a novel behavioral procedure to directly address this question.

Initially, using an operant two-chamber apparatus, the authors reproduce [4] that male rats (termed Observers) learn to chain-pull to release distressed conspecifics (termed Targets) from either a water-filled (distress condition) or an empty (e.g., no distress condition) chamber and subsequently interact in a dry compartment. Notably, when the authors reversed the role of each pair, such that the Observer became the Target and vice versa, new Observers were faster in releasing the new Target than the original ones. These data suggest that previous experience promotes faster acquisition of the task.

To show direct, rather than indirect, empathetic behavior in the absence of social interaction the authors added a third, physically separated but visually clear and perforated, compartment to their two-chamber apparatus. Here, contingent on the Observer chainpull, the distressed Target was rescued to the separate dry compartment in the absence of physical social interaction. Notably, Observers learned to aid conspecifics even without direct social interaction, although exclusively when Targets were in distress. This procedure is critically dependent on the presence of the distressed conspecific as evidenced by the failure of Observers to acquire stable responding for either an empty wet chamber or for an inanimate "fake rat". Using this novel procedure, Cox and Reichel extend previously established indirect empathetic behavioral procedures by removing direct physical social interaction as a potential confound.

Often conserved across species, many nonhuman animals and humans react to the affective state of others in a process coined "emotional contagion" [8]. Distressed individuals benefit from conspecific consolation behaviors, and conversely, a distressed individual can also transmit stress to others. Based on the bidirectional nature of emotional social transmission [8], the authors propose that the Targets' distress is "emotionally" transferred to the Observers to promote a similar affective state. Once the Observers perform their contingent response releasing the Targets, the consequent relief is also "emotionally" transferred and acts as a reinforcer to the Observers' actions.

This is a fascinating framework. However, previous findings show that rodents robustly and reliably self-administer a spectrum of socially reinforcing behaviors spanning from aggression [9] to the opportunity to gain access to a social peer [10]. Therefore, it is critical to determine the motivational contribution of direct social reinforcement relative to the direct empathic release a distressed conspecific. For this purpose, the authors increased the reinforcement schedule from a simple fixed-ratio (FR) 1 to more stringent FR5 and FR10 in both two- and three-chamber apparatus. Strikingly, Observers from the two-chamber apparatus, which allow direct social interaction, increased and maintained their responding once the required effort was increased. Observers from the three-chamber apparatus were significantly slower in releasing the distressed conspecifics, suggesting that direct empathic responding in the absence of social interaction is strongly modulated by effort. However, empathic responding was maintained over time as shown by the Observers' persistent release of distressed conspecifics for up to two weeks following the final day of acquisition. These findings, in agreement with previous literature $[3,4]$, support the notion that positive social interaction is critical in maintaining rats' empathetic behavior.

Finally, the authors show that the propensity to release a distressed conspecific is sensitive to previous familiarity with their paired conspecific. When a familiar Target is replaced with an unfamiliar Target in the water-filled pool, the Observer learns release the stranger in distress albeit with a significantly increased latency. These data indicate that rats can adjust their empathic behavior to help an unfamiliar, distressed conspecific. In combination, these data are remarkably intriguing. What neural

\footnotetext{
${ }^{1}$ Behavioral Neuroscience Branch Intramural Research Program, NIDA, NIH, Baltimore, USA and ${ }^{2}$ University of Washington, Department of Biological Structure, Seattle, USA Correspondence: Marco Venniro (venniro.marco@nih.gov) or Sam A. Golden (sagolden@uw.edu)

These authors contributed equally: Marco Venniro and Sam A. Golden
}

Received: 11 December 2019 Accepted: 16 December 2019

Published online: 2 January 2020 
mechanisms govern direct empathic behavior in rats? Are these a subset, or completely unique, from those governing direct social interaction? What social cues transfer direct empathic responding between familiar and non-familiar targets?

Cox and Reichel's novel rat direct empathy procedure is welltimed with the clear current interest in developing ethologically relevant behavioral procedures that allow researchers to better capture complex social behaviors [6]. This behavioral approach has direct applicability to myriad neuropsychiatric conditions, and it is with great interest and enthusiasm that behavioral neuroscientists await the mechanistic investigations that will take full advantage of this behavioral approach.

\section{FUNDING AND DISCLOSURE}

This work was supported by the Intramural Research Program of NIDA; fellowship from the NIH Center on Compulsive Behaviors (MV) and NIDA 4R00DA045662-02 (SAG). The authors declare no competing interests.

\section{ACKNOWLEDGEMENTS}

We thank Dr. Yavin Shaham and Dr. Leslie A. Ramsey for comments and edits on the early version of the paper.

\section{AUTHOR CONTRIBUTIONS}

M.V. and S.A.G. equally contributed to this manuscript.

\section{ADDITIONAL INFORMATION}

Publisher's note Springer Nature remains neutral with regard to jurisdictional claims in published maps and institutional affiliations.

\section{REFERENCES}

1. Church RM. Emotional reactions of rats to the pain of others. J Comp Physiol Psychol. 1959;52:132-4. https://doi.org/10.1037/h0043531.

2. Rice GE, Gainer P. "Altruism" in the albino rat. J Comp Physiol Psychol. 1962;55:123-5. https://doi.org/10.1037/h0042276.

3. Ben-Ami Bartal I, Decety J, Mason P. Empathy and pro-social behavior in rats. Science. 2011;334:1427-30. https://doi.org/10.1126/science.1210789.

4. Sato N, Tan L, Tate K, Okada M. Rats demonstrate helping behavior toward a soaked conspecific. Anim Cogn. 2015;18:1039-47. https://doi.org/10.1007/ s10071-015-0872-2.

5. Trezza V, Campolongo P, Vanderschuren LJ. Evaluating the rewarding nature of social interactions in laboratory animals. Dev Cogn Neurosci. 2011;1:444-58. https://doi.org/10.1016/j.dcn.2011.05.007.

6. Heilig M, Epstein DH, Nader MA, Shaham Y. Time to connect: bringing social context into addiction neuroscience. Nat Rev Neurosci. 2016;17:592-9. https:// doi.org/10.1038/nrn.2016.67.

7. Cox SS, Reichel CM. Rats display empathic behavior independent of the opportunity for social interaction. Neuropsychopharmacology. 2019. https://doi.org/ 10.1038/s41386-019-0572-8.

8. de Waal FBM, Preston SD. Mammalian empathy: behavioural manifestations and neural basis. Nat Rev Neurosci. 2017;18:498-509. https://doi.org/10.1038/ nrn.2017.72.

9. Golden $\mathrm{SA}$, et al. Compulsive addiction-like aggressive behavior in mice. Biol Psychiatry. 2017;82:239-48. https://doi.org/10.1016/j.biopsych.2017.03.004.

10. Venniro $M$, et al. Volitional social interaction prevents drug addiction in rat models. Nat Neurosci. 2018;21:1520-9. https://doi.org/10.1038/s41593-0180246-6. 